\title{
Realistic synthesis of brain tumor resection ultrasound images with a Generative Adversarial Network
}

\author{
Mélanie Donnez ${ }^{\mathrm{a}}$, François-Xavier Carton ${ }^{\mathrm{a}, \mathrm{b}}$, Florian Le Lann $^{\mathrm{c}}$, Emmanuel De Schlichting ${ }^{\mathrm{c}}$, \\ and Matthieu Chabanas ${ }^{a}$ \\ ${ }^{a}$ University of Grenoble Alpes, CNRS, Grenoble-INP, TIMC-IMAG; Grenoble, France \\ ${ }^{b}$ Dept. of Electrical Engineering and Computer Science, Vanderbilt University, Nashville, USA \\ ${ }^{\mathrm{c}}$ Grenoble Alpes University Hospital, Department of Neurosurgery; Grenoble, France
}

\begin{abstract}
The simulation of realistic ultrasound (US) images has many applications in image-guided surgery such as image registration, data augmentation, or educational purposes. In this paper we simulated intraoperative US images of the brain after tumor resection surgery. In a first stage, a Generative Adversarial Networks generated an US image with resection from a resection cavity map. While the cavity texture can be realistic, surrounding structures are usually not anatomically coherent. Thus, a second stage blended the generated cavity texture into a real patient-specific US image acquired before resection. A validation study on 68 images of 21 cases showed that three raters correctly identified $64 \%$ of all images. In particular, two neurosurgeons correctly labelled only $56 \%$ and $53 \%$ of the simulated images, which indicate that these synthesized images are hardly distinguishable from real post-resection US images.
\end{abstract}

Keywords: Intraoperative Ultrasound, Image synthesis, Generative Adversarial Networks, Neurosurgery.

\section{INTRODUCTION}

Intraoperative ultrasound (iUS) is commonly used during brain tumor resection to localize tissue and ensure than the tumor removal is as complete as possible. These iUS images can be used as is in neuronavigation systems, or could also be registered with preoperative Magnetic Resonance (MR) images to compensate for brain-shift deformation. ${ }^{1}$

The simulation of realistic iUS images, especially after tumor resection, is valuable in several applications such as surgical planning or medical training, to shorten the learning curve of young surgeons. ${ }^{2}$ Another potential application is the registration of preoperative MR images with intraoperative iUS images at the end of tumor resection, to guarantee that tumor tissue was optimally removed. Instead of multimodal registration, always a challenging task, one strategy is to simulate US images from the preoperative MR then perform monomodal registration between these simulated US and the actual iUS images. ${ }^{3}$ However, this monomodal registration is still hindered by the fact that the resection cavity is visible in the real iUS images only. Simulating US images with a resection cavity, which would follow the tumor contours delineated in the MR, is expected to significantly simplify this registration problem. Finally, another targeted application is data augmentation to train deep neural networks. We recently proposed such a network to automatically segment resection cavities in iUS images. ${ }^{4}$ However, realistically simulating a large number of resection patterns would enable to enlarge the training set and thus improve the robustness of the segmentation network.

Many authors proposed to simulate the physics of ultrasonic waves propagation and backscattering on biological tissue, ${ }^{3,5,6}$ typically to generate US images from CT or MR volumes. More recently, several groups used deep neural networks to simulate intravascular or kidney US images. ${ }^{2,7}$ These works especially used Generative Adversarial Networks ${ }^{8}$ (GAN) which consist in two neural networks: a generator learns while attempting to generate realistic ultrasound images, while a discriminator simultaneously learns while attempting to discriminate between real images and those simulated by the generator. These networks are typically trained with real US images along with segmented tissue-maps of anatomical structures. Other tissue-maps can then be input into the trained GAN to synthesize the corresponding US image. 
In many contexts the same anatomical structures are visible in all images (for instance the kidney, spleen, and bones in Pigeau et al. ${ }^{2}$ ), which differ only due to anatomical variations and pathology, and image characteristics. A major challenge in our context is that intraoperative US images of the brain are extremely different from patient to patient: due to the variation of tumor locations, no common structures can be reliably found in the iUS images. Sulci are almost always present, but their number and shape also depend on the imaged area. For these reasons, learning the relations between iUS images and tissue-maps only seemed extremely challenging.

The proposed method simulates post-resection iUS images of the brain from two inputs: a patient-specific iUS image acquired before resection and a resection cavity map. This is realized in two stages: 1) a GAN network generates a pseudo iUS image from a resection cavity map. While the cavity texture can be realistic, surrounding structures usually do not correspond to coherent anatomical features; 2) the GAN-generated cavity texture is then merged into a real iUS image acquired before resection to simulate the final image. A validation study was carried out with three raters, including two neurosurgeons, to evaluate whether they could distinguish simulated images from real ones. In this study, we used cavity maps segmented from real post-resection iUS images, so that the synthesized images can be directly compared with these real images.

\section{MATERIALS AND METHODS}

\subsection{Dataset}

We used intraoperative ultrasound images of patients with low-grade gliomas from the public database RESECT. ${ }^{9}$ 21 of the 23 cases of the database could be processed in this study, with two 3D volumes per case: iUS before

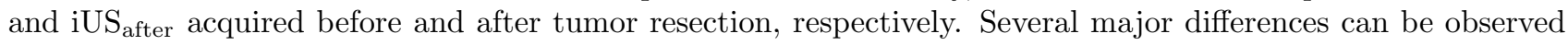
between these two volumes:

- the US probe position changed between the two acquisitions yielding to different fields of view, image textures (intensities, speckle, noise), and potentially different reconstruction artefacts.

- the brain-shift phenomenon induces tissue deformation during surgery, due to several factors like gravity, loss of Cerebrospinal fluid, or drugs. Thus, anatomical structures in the two volumes do not exactly fit.

- in the iUS ${ }_{\text {after }}$ volume, the tumor was removed and a resection cavity is instead visible. This cavity can induce additional tissue deformation as well as artifacts in the images (typically a hyper-intense area at the bottom of the cavity and ultrasound shadows). The cavity borders could also be appear brighter due to bleeding.

To compensate for part of the brain-shift, $\mathrm{iUS}_{\text {before }}$ and $\mathrm{iUS}_{\text {after }}$ volumes were non-rigidly registered with thin plate splines between homologous landmarks available in the RESECT database. Overall, the mean distance between landmarks (i.e. the fiducial registration error) was reduced from $3.55 \pm 1.76$ to basically zero. $18572 \mathrm{D}$ images containing a tumor were extracted from these $3 \mathrm{D}$ volumes, and later used in this study.

To simulate complete tumor resections, we used the real resection cavity masks segmented from the iUS $_{\text {after }}$ images. ${ }^{4}$ Examples of registered iUS before $_{\text {and }}$ iUS $_{\text {after }}$ images as well as resection cavity masks are available in figure 3.

\subsection{Stage I: GAN simulation of a resection cavity image}

The pix2pix conditional GAN developed by Isola et al. ${ }^{8}$ was used as pictured in figure 1 . The generator simulates an US image with resection from a resection cavity map, while the discriminator compares this image to the

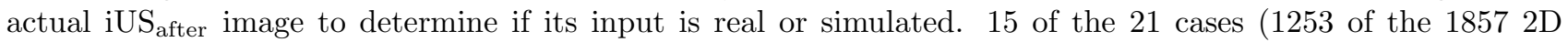
images) were used for training, plus 1 case $(108$ images) for validation. The remaining images were kept for testing.

After this first stage, the GAN-generated image contains a realistic resection cavity. However its surrounding looks like an iUS image except that the structures (sulci, ventricles...) may not be anatomically coherent. 


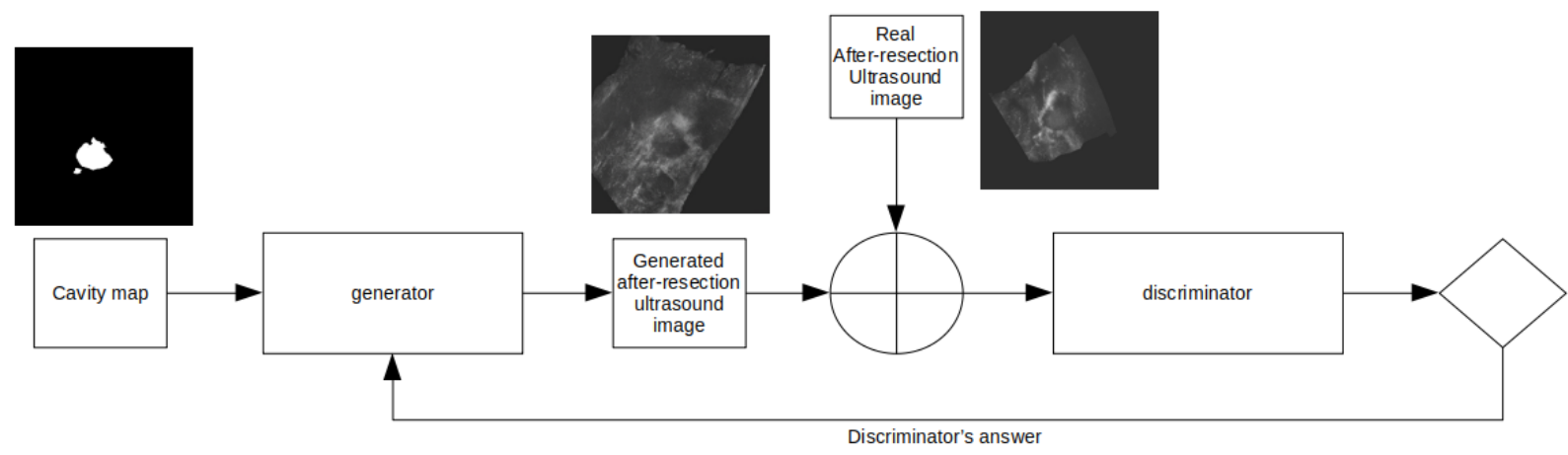

Figure 1. Stage I: GAN generation of an iUS with resection from a cavity map and real after-resection iUS images.

\subsection{Stage II: simulated image in a patient's context}

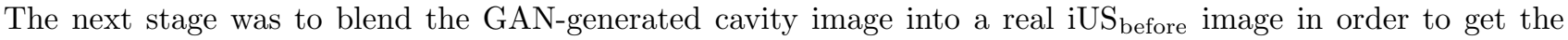
cavity within a patient's anatomical features and thus simulate a realistic iUS image after resection.

Intensities of the two images to blend may significantly differ. Therefore, a first step was to normalize the GAN-generated resection image by equalizing its histogram to the histogram of the iUS before $_{\text {image. Histograms }}$ were computed on a region of interest (ROI) centered on the cavity, the ROI's size being twice the size of the cavity.

After this normalization, pixels of the simulated image were assigned as follows:

- if a pixel is located inside the cavity map, its intensity is taken from the GAN-generated resection image;

- if a pixel is outside the cavity map but at a distance $d$ inferior to half the cavity size, the pixel intensity is linearly interpolated from the two images with a weighting factor depending on $d$ (the closest image has more influence);

- if a pixel is outside this blending area, its intensity is taken from the iUS $\mathrm{Sefore}_{\text {image. }}$

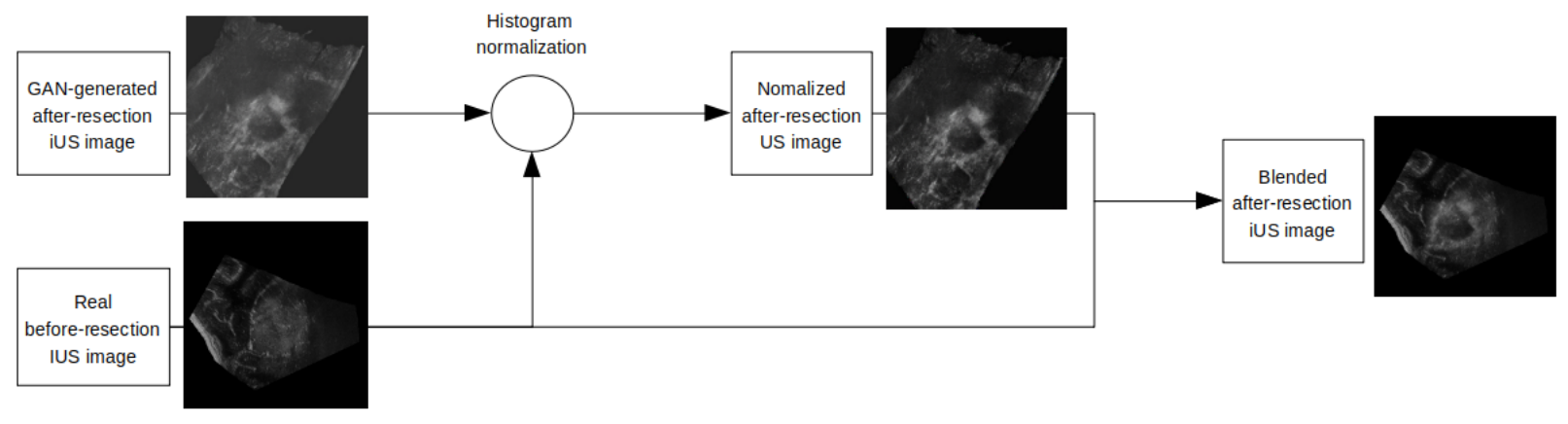

Figure 2. Stage II: merging of the GAN-generated resection cavity image into a real before-resection iUS image.

\subsection{Validation}

Three raters participated in the validation study, a medical imaging expert (rater 1) and two neurosurgeons who have routinely used intraoperative US during tumor resection. Although none of the raters was involved in the methodological developments of this paper, raters 1 and 2 already worked on the RESECT database to manually 
segment resection cavities in iUS images. ${ }^{4}$ Despite this bias, these two raters were still included in the validation study as they worked on the images more than a year ago and as they never saw any simulated iUS image.

The first validation test was a form of Visual Turing Test used in Pigeau et al. ${ }^{2}$ to evaluate how well a person can differentiate between real and synthesized images, thus determining how convincing the synthesized images are. The dataset consisted in 68 2D images, 34 simulated and 34 real ones, selected from all cases and presented in a random order. Each rater had to label each image as simulated or real, and score his confidence in his choice on a scale of 1 to 5 .

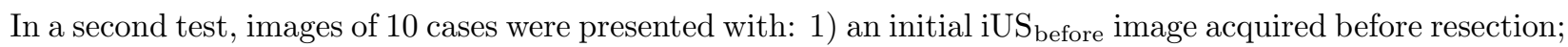
2) a resection cavity mask (segmented from the fourth image); 3) the image simulated from these inputs; 4) and the corresponding real $\mathrm{iUS}_{\text {after }}$ image acquired after resection. Examples of these images are shown in figure 3. Note that the simulated and real iUS images after resection are always different, because the simulation was based on the image acquired before resection. The goal here was simply to qualitatively determine whether the simulated images were plausible, and the aspects that were correctly simulated or could be improved.

\section{RESULTS AND DISCUSSION}

Examples of GAN-generated resection and final simulated images are shown on figures 1 and 3, respectively. Results of the differentiation study are presented in table 1.

If real and simulated images were easy to distinguish, $100 \%$ of the images should be correctly labelled. Conversely, a score of $50 \%$ would mean that there is no way to discriminate real from synthesized images, suggesting simulated images appear realistic. Overall, $64 \%$ of the images were correctly labelled. Significant differences can be observed among raters: rater 1 who extensively manually segmented structures on the RESECT images could distinguish almost $80 \%$ of all images. However, only $56 \%$ and $53 \%$ of the simulated images were labelled as such by the two neurosurgeons which is an excellent result. Cases that were used in the training phase of the GAN network were more difficult to discriminate that test cases, which suggests the cavity appearance of new cases is slighlty less realistic.

\begin{tabular}{lcccc}
\multicolumn{5}{c}{ Table 1. Percentages of correctly labelled images. } \\
\hline & $\begin{array}{c}\text { Rater 1 } \\
\text { Imaging expert }\end{array}$ & $\begin{array}{c}\text { Rater 2 } \\
\text { Neurosurgeon }\end{array}$ & $\begin{array}{c}\text { Rater 3 } \\
\text { Neurosurgeon }\end{array}$ & All raters \\
\hline Simulated images & 0.79 & 0.56 & 0.53 & 0.63 \\
train cases (GAN stage) & 0.78 & 0.48 & 0.54 & 0.59 \\
$\quad$ test cases (GAN stage) & 0.82 & 0.72 & 0.52 & 0.69 \\
Real images & 0.76 & 0.79 & 0.41 & 0.65 \\
Overall & 0.78 & 0.68 & 0.47 & 0.64 \\
\hline Confidence score & $2.74 \pm 1.2$ & $3.37 \pm 0.8$ & $3.32 \pm 1.34$ & $3.14 \pm 1.2$ \\
\hline
\end{tabular}

The qualitative analysis of 10 cases revealed that all raters were globally very satisfied with the simulated images. Several limits were nevertheless identified, mostly than the resection cavity margins can appear blurry when the texture of the GAN-generated cavity significantly differs from the iUS before texture. Also, images may lack variability since several features that could be observed in real images are never synthesized, like high intensity areas from blood clots on the resection cavity borders, or deep shadows below the cavity.

\section{CONCLUSION}

We simulated post-resection iUS images by generating an image of a resection cavity with a GAN network, then merging this image into a real patient-specific iUS image before resection. Despite blurriness on some cases, validation results showed than most simulated images were hardly distinguishable from real images.

Future development will be to include the iUS before image as a GAN input, along with the cavity map, to generate resection images which intensity, texture, and speckle better match the patient image characteristics. Also, we plan to evaluate the simulation of iUS images from more anatomical maps of sulci, ventricles or the tumor, and directly from preoperative MR images. 

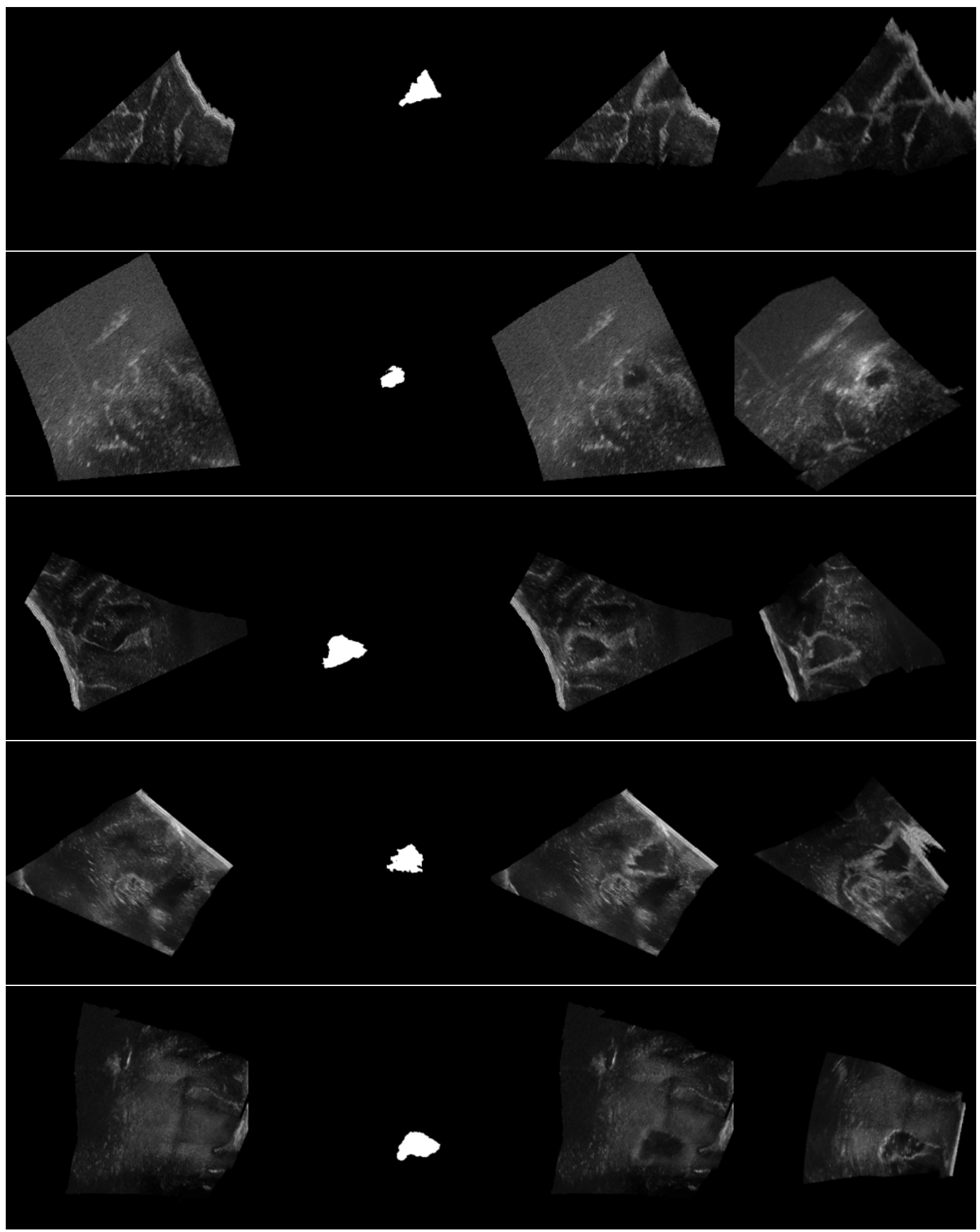

Figure 3. Images of 5 cases. From left to right: 1) the initial iUS image acquired before resection; 2) a resection cavity mask; 3) the result image simulated from these inputs; and 4) the corresponding real iUS image acquired after resection. In this study, resection cavity masks (column 2) correspond to the actual resection cavities segmented in the real iUS image after resection (column 4). 


\section{ACKNOWLEDGMENTS}

This work was supported by the French National Research Agency (ANR) through the frameworks Investissements d'Avenir Labex CAMI ANR-11-LABX-0004, ANR-15-IDEX-02, and MIAI ANR-19-P3IA-0003. The GAN computations were performed using the GRICAD infrastructure (gricad.univ-grenoble-alpes.fr), which is supported by Grenoble research communities.

\section{REFERENCES}

[1] Xiao, Y., Rivaz, H., Chabanas, M., Fortin, M., Machado, I., Ou, Y., Heinrich, M., Schnabel, J. a., Zhong, X., Maier, A., Wein, W., Shams, R., Kadoury, S., Drobny, D., Modat, M., and Reinertsen, I., "Evaluation of mri to ultrasound registration methods for brain shift correction: The curious2018 challenge," IEEE Transactions on Medical Imaging 39(3), 777-786 (2020).

[2] Pigeau, G., Elbatarny, L., Wu, V., Schonewille, A., Fichtinger, G., and Ungi, T., "Ultrasound image simulation with generative adversarial network," in [SPIE Medical Imaging 2020: Image-Guided Procedures, Robotic Interventions, and Modeling], 11315, 54-60 (2020).

[3] Mercier, L., Fonov, V., Haegelen, C., Del Maestro, R. F., Petrecca, K., and Collins, D. L., "Comparing two approaches to rigid registration of three-dimensional ultrasound and magnetic resonance images for neurosurgery," International journal of computer assisted radiology and surgery 7(1), 125-136 (2012).

[4] Carton, F.-X., Chabanas, M., Le Lann, F., and Noble, J. H., "Automatic segmentation of brain tumor resections in intraoperative ultrasound images using u-net," Journal of Medical Imaging 7(3), 1 - 15 (2020).

[5] Bamber, J. C. and Dickinson, R. J., "Ultrasonic b-scanning: a computer simulation," Physics in Medicine and Biology 25(3), 463-479 (1980).

[6] Karamalis, A., Wein, W., and Navab, N., "Fast ultrasound image simulation using the westervelt equation," in [Medical Image Computing and Computer-Assisted Intervention (MICCAI)], (2010).

[7] Tom, F. and Sheet, D., "Simulating patho-realistic ultrasound images using deep generative networks with adversarial learning," in [IEEE 15th Int. Symposium on Biomedical Imaging (ISBI 2018)], 1174-1177 (2018).

[8] Isola, P., Zhu, J.-Y., Zhou, T., and Efros, A. A., "Image-to-image translation with conditional adversarial networks," in [IEEE Conference on Computer Vision and Pattern Recognition (CVPR)], (2017).

[9] Xiao, Y., Fortin, M., Unsgård, G., Rivaz, H., and Reinertsen, I., "Retrospective evaluation of cerebral tumors (resect): A clinical database of pre-operative mri and intra-operative ultrasound in low-grade glioma surgeries," Medical Physics 44(7), 3875-3882 (2017).

\section{THIS WORK HAS NOT BEEN SUBMITTED ELSEWHERE}

